\title{
Les marges de raffinage : évolution récente
}

\author{
C. Baudouin ${ }^{1}$ et J.-P. Favennec ${ }^{1}$ \\ 1 Institut français du pétrole, 1 et 4, avenue de Bois-Préau, 92852 Rueil-Malmaison Cedex - France \\ e-mail : colin.baudouin@ifp.fr - i-pierre.favennec@ifp.fr
}

\begin{abstract}
Résumé - Malgré une conjoncture globalement médiocre, du fait notamment de la crise asiatique et d'un hiver clément dans l'hémisphère nord, l'année 1997 a confirmé les signes d'amélioration de l'activité du raffinage observés en 1996. Mais la situation de ce secteur n'est pas encore satisfaisante : faible rentabilité des capitaux employés, préoccupations quant aux financements des investissements nécessaires à la protection de l'environnement. Pour 1998, la chute des cours des pétroles bruts, qui a évidemment pour corollaire une baisse des prix des produits, a été finalement plutôt favorable aux marges. Deux éléments modèrent ce relatif optimisme : d'une part le maintien d'une très grande volatilité, d'autre part l'accentuation de la crise économique et financière observée au cours de l'été, qui s'est traduite par une forte chute des marges dans toutes les zones géographiques et plus particulièrement en Asie. Début 1999, les marges de raffinage sont restées faibles et ont conduit à une baisse du taux d'utilisation des capacités de traitement.
\end{abstract}

Mots-clés : marges de raffinage, Europe, spécifications, pétrole brut, évolution.

\begin{abstract}
Refining Margins: Recent Trends - Despite a business environment that was globally mediocre due primarily to the Asian crisis and to a mild winter in the northern hemisphere, the signs of improvement noted in the refining activity in 1996 were borne out in 1997.

But the situation is not yet satisfactory in this sector: the low return on invested capital and the financing of environmental protection expenditure are giving cause for concern. In 1998, the drop in crude oil prices and the concomitant fall in petroleum product prices was ultimately rather favorable to margins.

Two elements tended to put a damper on this relative optimism. First of all, margins continue to be extremely volatile and, secondly, the worsening of the economic and financial crisis observed during the summer made for a sharp decline in margins in all geographic regions, especially Asia.

Since the beginning of 1999, refining margins are weak and utilization rates of refining capacities have decreased.

Keywords: Refining margin, Europe, specifications, crude oil, trend.
\end{abstract}

\section{LE CONTEXTE DU RAFFINAGE : LA DEMANDE DE PRODUITS - L'OFFRE DE PÉTROLE BRUT}

La consommation mondiale de produits pétroliers (fig. 1) a augmenté de 2,3\% en 1997 pour atteindre 73,4 millions de barils par jour (b/j). En Asie, la hausse a été nettement plus marquée et a atteint 5,6\% (7,5\% si on inclut la Chine). À l'inverse, la demande en Europe ne s'est accrue que faiblement, d'environ $1 \%$, malgré une certaine reprise économique. Mais l'hiver 1997 a été très doux comparé au précédent. Aux États-Unis et en Amérique latine, les consommations ont progressé aux alentours du rythme mondial.

En 1998, la consommation mondiale s'est établie à 73,7 millions de b/j et n'a augmenté que de 0,5\%. En Asie, la crise a stoppé net la progression de la consommation de la région, même si deux "poids lourds", la Chine et l'Inde, ne semblent pas affectés pour l'instant par les difficultés de leurs voisins et connaissent encore une croissance robuste de leur demande en pétrole. Deux autres régions du monde ont également été durement touchées par des crises économiques, 
avec des conséquences différentes sur leur consommation pétrolière. En Russie, la crise s'est traduite par une forte diminution de la consommation $(-1,7 \%)$. En revanche, la crise monétaire au Brésil n'a pas empêché une croissance soutenue de la consommation en Amérique latine (+3,6\%). Dans ce contexte international défavorable, les États-Unis ont vu leur consommation n'augmenter que de 1,2\%. L'Europe a, pour sa part, su profiter de sa bonne santé économique, avec une hausse de sa consommation de pétrole de 1,4\%.

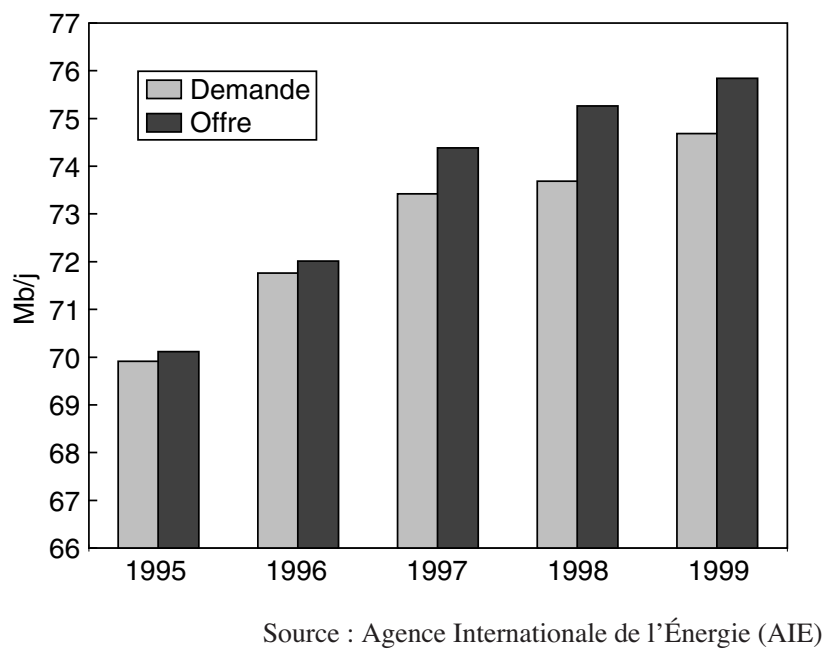

Figure 1

Évolution de l'offre et de la demande mondiale de pétrole. Evolution of the supply and demand of oil.

L'offre de pétrole est très abondante. Elle a crû de 3,3\% en 1997 et de 1,2\% en 1998, donc sensiblement plus vite que la demande. Il en est résulté un accroissement important des stocks de bruts et de produits, qui est l'une des principales causes de la baisse du prix du brut. À court terme, le potentiel d'augmentation de la production aussi bien dans les pays Opep que non-Opep reste très important.

En outre, les découvertes récentes (Brésil, Congo, Nigeria, Angola, golfe du Mexique et même mer du Nord) correspondent à des bruts relativement légers et de teneur en soufre modérée, ce qui éloigne un peu plus l'horizon d'une pénurie de brut léger de bonne qualité.

\section{LES MARGES DE RAFFINAGE (Fig. 2)}

En Europe, les marges de raffinage à Rotterdam se sont stabilisées entre 1 et $2 \$ / b$ (sur craquage catalytique ; notation CC) sur la période 1997-1998, grâce notamment aux exportations d'essences vers les États-Unis. En aoûtseptembre 1998, la situation a évolué dans un sens un peu moins favorable, avec une baisse de la marge de plus de $1 \$ / \mathrm{b}$ par rapport aux mois précédents. Depuis, la situation s'est un peu améliorée.

\section{Marges et prix du brut}

$\mathrm{Si}$ les marges de raffinage restent acceptables alors que le prix du brut est au plus bas, c'est que l'écart entre celui-ci et ceux des produits pétroliers leur demeure favorable. Rappelons que le calcul des marges brutes de raffinage est la différence entre la valorisation des produits obtenus (dans des proportions déterminées) et le coût d'achat du pétrole brut. Pour le calcul des marges nettes, il convient de soustraire les frais variables aux marges brutes. Par conséquent, si l'écart entre les prix des produits pétroliers et du pétrole brut est relativement stable, les marges restent équilibrées.

De même, l'amélioration des résultats de la distribution en France est liée à une baisse modérée du prix des carburants par rapport à celle du prix du brut, toutes choses (i.e. les taxes) égales par ailleurs. Autrement dit, les sociétés pétrolières peuvent compenser par ce biais une partie de leur perte financière amont.

Aux États-Unis, le manque de flexibilité (production maximale d'essences) et l'utilisation massive des capacités de raffinage limitent les réactions possibles aux nombreuses variations de la demande (demande de distillats en augmentation, durcissement des spécifications et nouveaux carburants, brut raffiné plus lourd, etc.). Toutefois, la baisse des prix et le surplus d'offre de brut ont permis aux raffineurs américains en 1997 d'obtenir des marges satisfaisantes et supérieures à celles des années précédentes $(1,46 \$ / \mathrm{b}$ en 1997 pour les marges du golfe du Mexique, soit le double par rapport à 1996). Néanmoins, un hiver doux et une réduction du différentiel essence/brut expliquent leur chute au quatrième trimestre de l'année 1997.

Au printemps 1998, les marges sont redevenues très satisfaisantes. Mais dès le mois d'août, elles se sont à nouveau fortement dégradées. Elles sont même devenues négatives les deux derniers mois de l'année en raison d'hivers relativement doux, de stocks élevés en distillats et en essences, et du prix des essences à leur plus bas niveau depuis plus de huit ans. Au bilan, la marge CC sur le brut West Texas Intermediate (WTI) s'est établie à $1,22 \$ / b$ en 1998 . Le résultat n'est donc pas foncièrement mauvais et finalement peu éloigné de celui de 1997. En fait, le point le plus important depuis deux ans dans cette région reste la volatilité des marges qui ne cesse de s'accentuer. Enfin, il est important de souligner l'écart très sensible entre les marges du golfe du Mexique et celles observées à l'intérieur du continent américain ou en Californie qui sont nettement supérieures et peuvent atteindre 5 ou $6 \$ / b$.

Les marges en Asie sont par contre tombées à des niveaux très bas, tant du fait de la faiblesse de la consommation (en dehors de la Chine) que de l'apparition de surcapacités de 

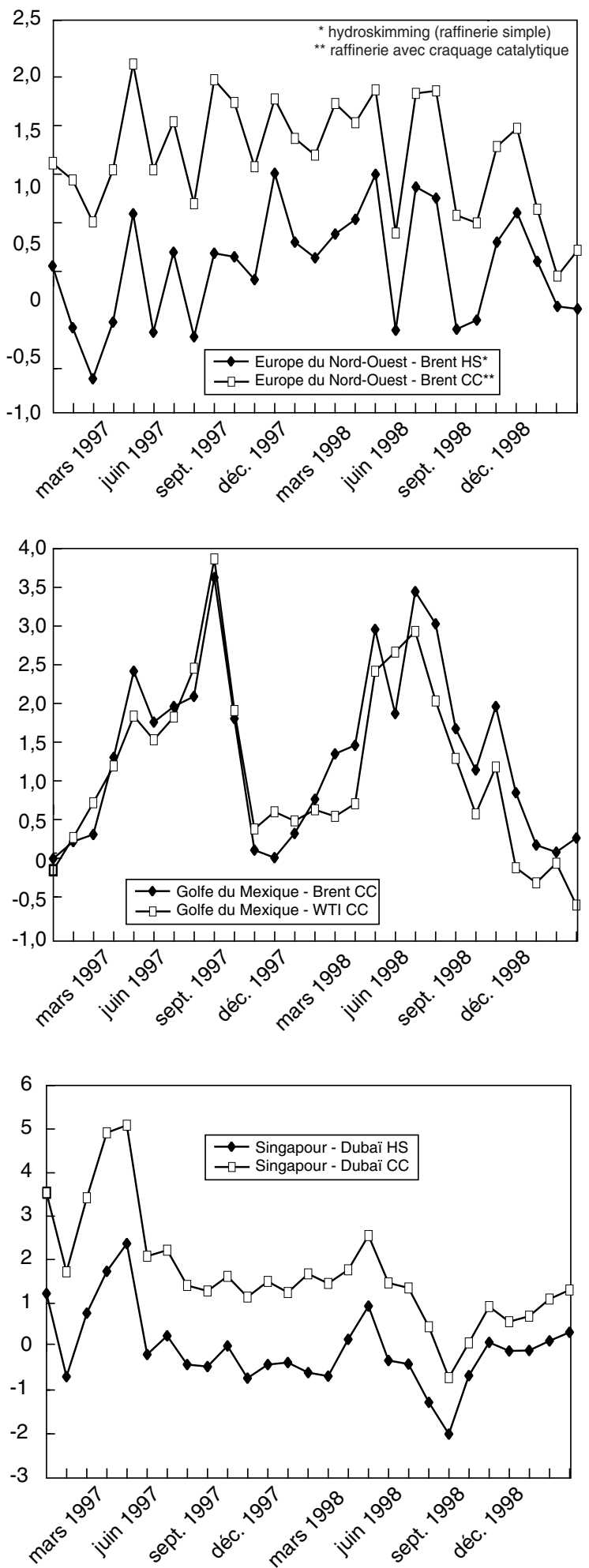

Source : Agence Internationale de l'Énergie

Figure 2

Évolution des marges de raffinage en Europe, aux États-Unis et en Asie (\$/b).

Evolution of refining margins in the Europe, United States and in Asia $(\$ / b)$. raffinage. Alors qu'elles ont augmenté de $720000 \mathrm{~b} / \mathrm{j}$ soit 36 millions de tonnes par an (Mt/an) en 1997, les capacités de raffinage inutilisées dans cette région à la mi-1998 sont estimées à près de $1 \mathrm{Mb} / \mathrm{j}$. En outre, la volonté des autorités chinoises d'accroître le taux de marche des raffineries du pays les a conduites à importer davantage de brut et moins de produits, aggravant ainsi les excédents de produits dans une zone qui naguère était encore déficitaire. En 1998, la consommation de produits pétroliers en Asie (y compris Chine, Japon et Corée du Sud) a diminué de 420000 b/j. L'avenir des marges dans cette région reste donc sombre.

\section{LA SURCAPACITÉ DE TRAITEMENT EN EUROPE}

Problème récurrent du raffinage européen, la surcapacité de traitement est toujours évaluée par certains à 60 Mt de distillation (une dizaine au Royaume-Uni, une vingtaine dans le Bénélux et une trentaine en Italie), équivalant à la fermeture de 10 à 15 raffineries. Pour une consommation de produits pétroliers de l'ordre de $580 \mathrm{Mt} / \mathrm{an}$, l'Union européenne dispose d'une capacité de distillation supérieure à $655 \mathrm{Mt} / \mathrm{an}$ et ne traite que $600 \mathrm{Mt} / \mathrm{an}$ environ.

Au niveau européen, trois régions semblent particulièrement touchées par le problème de la surcapacité.

- En Bavière, la production est élevée alors que les possibilités d'exportations sont faibles. La surcapacité dans cette région est importante. En outre, l'ouverture de la raffinerie de Leuna en Saxe Anhalt (capacité de 8,7 Mt/an, en remplacement d'une raffinerie de $5 \mathrm{Mt} / \mathrm{an}$ ) pourrait accentuer ce déséquilibre. Toutefois, le regroupement de quatre compagnies bavaroises au sein d'une association, nommée Bayernoil, laisse envisager une réduction rationnelle des capacités.

- Au Royaume-Uni, un outil de raffinage sophistiqué, la production de la mer du Nord à proximité et les exportations d'essences vers les États-Unis expliquent la difficulté d'atténuer les surcapacités de production. De plus, la fermeture de nombreuses stations-service a entraîné une remise à niveau des marges de distribution après la guerre des prix de 1996. Enfin, signalons que la seule fermeture prévue, qui concerne le site de Shell à Haven, ne doit intervenir que fin 1999. Par ailleurs, la raffinerie de Gulf à Haven, fermée depuis un an, risque d'être vendue (à Petroplus International) et donc réutilisée.

- Dans le sud-est de la France, la région de Berre-Marseille regroupe presque $30 \mathrm{Mt} / \mathrm{an}$ de capacités de traitement et est nettement exportatrice. Mais contrairement à ce qui s'est passé en Bavière, les raffineurs n'ont pas réussi à s'accorder pour réduire les capacités. De plus, trois des quatre raffineries concernées sont fortement intégrées en aval dans la pétrochimie ce qui améliore leur rentabilité. 


\section{Le cas français}

En 1997, le secteur du raffinage et de la distribution en France a dégagé un bénéfice après impôts de 3,2 milliards de francs, contre 470 millions en 1996 et des pertes en 1994 et en 1995. Ce bon résultat s'explique, malgré une demande intérieure stagnante, par :

- une hausse du volume traité (plus 5,6\% par rapport à 1996);

- une amélioration conjoncturelle de la marge de raffinage (113 F/t contre $101 \mathrm{~F} / \mathrm{t}$ en 1996), en partie due à l'appréciation du dollar par rapport au franc ;

- des gains de productivité (réduction des frais fixes).

Différents éléments sont cependant fréquemment avancés pour justifier la nécessité de restructurations :

-le taux de rendement des capitaux employés, de l'ordre de $4,4 \%$, reste faible. Pour obtenir un taux plus satisfaisant (8 \%) il faudrait une marge de 130/140 F/t, soit environ $3 \$ / \mathrm{b}$;

- les frais de fabrication en France sont les plus élevés d'Europe (environ $11 \%$ de plus que la moyenne européenne, soit $13 \mathrm{~F} / \mathrm{t}$ ) ;

-le taux de conversion $(24,7 \%)$ est sensiblement inférieur à celui des autres pays européens, alors que les excédents de fioul lourd à haute teneur en soufre (HTS) continuent de croître ;

- la demande d'essences est relativement faible et des excédents importants (3 Mt) doivent être exportés. Inversement, en raison d'une consommation de gazole très soutenue, la France a dû importer 8 Mt de ce produit en 1997 ;

- les investissements à effectuer en France pour faire face au durcissement des spécifications européennes pourraient s'élever à 13 milliards de francs entre 1998 et 2010, soit un milliard par raffinerie française ;

- la lutte contre l'effet de serre pèsera sur le raffinage dans la mesure où les émissions de $\mathrm{CO}_{2}$ devraient augmenter pour satisfaire l'évolution de la qualité des produits.

Malgré cela, l'incertitude sur le nombre exact de fermetures à court et à moyen terme demeure, et ce pour plusieurs raisons :

- les États-Unis restent toujours une zone importatrice d'essences ;

- le taux de marche des raffineries françaises est supérieur à $95 \%$;

-les investissements les plus massifs requis pour le durcissement des spécifications européennes sont, en pratique, reportés à l'horizon 2005 ;

- le coût de fermeture (y compris dépollution du site) d'une raffinerie reste dissuasif (plusieurs centaines de millions de francs pour une raffinerie moyenne);

- la réduction du différentiel de taxes entre gazole et essence pourrait avoir un impact sur les consommateurs et permettre un rééquilibrage progressif de la demande (plus d'essence et moins de gazole).

Jusqu'à ce jour, les réductions de capacités fréquemment "réclamées" ou suggérées ne se sont pas traduites dans les faits. Pour les trois régions évoquées précédemment, une réduction globale d'une dizaine de millions de tonnes semble déjà constituer un maximum. S'il est clair que les taux de marche restent modérés (environ $90 \%$ alors que des taux de 95 à $98 \%$ sont sans doute réalisables), aucun raffineur n'est prêt à supporter seul des coûts de fermeture importants, sans aucune garantie d'assister à une amélioration de la situation. Le risque de voir des concurrents tenter de prendre la place libre ou les importations s'accroître est loin d'être négligeable. En outre, la légère amélioration des marges depuis deux ans est un signe encourageant et ce, d'autant plus que les gains de productivité (donc les réductions de coûts) sont impressionnants.

L'alliance BP-Mobil (96-97) et les dernières annonces de restructuration faites par Shell vont dans ce sens. Plus globalement, l'industrie pétrolière voit se concrétiser des projets de mégafusion (BP-Amoco,-Arco Exxon-Mobil, TotalPetrofina), dont un des objectifs est clairement l'accroissement de la rentabilité du raffinage.
D'ici à l'an 2000, peu de bouleversements sont à attendre en matière de capacités en Europe. D'ailleurs, comme le montre le tableau 1 , les capacités pourraient même augmenter légèrement.

\section{DES CONTRAINTES RENFORCÉES APRÈS L'AN 2000 SUR LE RAFFINAGE EUROPÉEN}

À la suite de l'accord intervenu le 29 juin 1998 entre le conseil des ministres des Quinze et le Parlement européen, les nouvelles normes européennes pour les carburants ont été adoptées. La spécification la plus contraignante est la teneur maximale en soufre des essences automobiles et du gazole moteur qui sera, pour l'an 2000, de $150 \mathrm{ppm}$ et de $350 \mathrm{ppm}$, contre 500 ppm actuellement. Pour l'an 2005, cette teneur sera obligatoirement ramenée à $50 \mathrm{ppm}$ pour les deux carburants. Les pays d'Europe du Sud, qui ont un approvisionnement en pétrole brut plus lourd et plus soufré que la moyenne européenne, pourront bénéficier de dérogations. Quoi qu'il en soit, il est important de constater que les limites imposées correspondent 
TABLEAU 1

Changements de capacités en Europe en Mt (fin 1996/fin 1998)

Changes in refining capacities in Europe - Mt/y (end 1996/end 1998)

Additions de capacités

\begin{tabular}{|c|c|c|c|c|c|}
\hline Pays & Lieu & Opérateur & Capacité & & \\
\hline Allemagne & Leuna & Elf & 10,61 & Ouverture & 97 \\
\hline Allemagne & Vohburg/IN & Bayernoil & 0,92 & Extension & 97 \\
\hline Allemagne & Wilhelmshaven & Beta WRG & 2,23 & Extension & 98 \\
\hline Allemagne & Wesseling & $D E A M A G$ & 0,99 & Extension & 98 \\
\hline France & La Mède & Total & 0,90 & Extension & 97 \\
\hline France & Gonfreville & Total & 5,59 & Redémarrage & 97 \\
\hline France & Lavera & $B P$ & 0,52 & Extension & 98 \\
\hline Royaume-Uni & Haven & Petroplus & 5,55 & Rachat? & 99 \\
\hline Espagne & S. Vizcaya & Petronor SA & 0,55 & Extension & 98 \\
\hline Royaume-Uni & Pembroke & Texaco & 0,50 & Extension & 98 \\
\hline Royaume-Uni & Grangemouth & $B P$ & 0,56 & Extension & 98 \\
\hline Belgique & Antwerp & Fina & 0,99 & Extension & 98 \\
\hline Divers* & - & - & 2,85 & Extension & 98 \\
\hline Total & & & $32,76^{* *}$ & & \\
\hline
\end{tabular}

Réductions de capacités

\begin{tabular}{|c|c|c|c|c|c|}
\hline Pays & Lieu & Opérateur & Capacité & & \\
\hline Royaume-Uni & Haven & Shell & 4,56 & Fermeture & 99 \\
\hline Allemagne & Karlsruhe & Mineral & 2,02 & Réduction & 97 \\
\hline Allemagne & Leuna & Leuna & 4,96 & Fermeture & 97 \\
\hline Allemagne & Schwedt & $P C K$ & 0,99 & Réduction & 98 \\
\hline France & Reichstett & Shell & 3,97 & Fermeture? & 02 \\
\hline France & Berre & Shell & 2,30 & Réduction & 98 \\
\hline Danemark & Stignaes & $K P C$ & 2,85 & Fermeture & 97 \\
\hline Royaume-Uni & Haven & Gulf & 5,55 & Fermeture & 97 \\
\hline Pays-Bas & Europoort & $N R C$ & 3,50 & Fermeture & 98 \\
\hline Italie & Busalla & Iplom SpA & 0,64 & Réduction & 98 \\
\hline Divers* & - & - & 1,06 & Réduction & 98 \\
\hline Total & & & 32,40 & & \\
\hline
\end{tabular}

** À ce chiffre, il convient peut-être de rajouter 9,5 Mt pour la raffinerie de Milazzo en Italie (source Oil \& Gas Journal qui vient, par ailleurs, d'insérer dans sa base de données la raffinerie d'Universal à Anvers en Belgique, d'une capacité de 3,22 Mt).

TABLEAU 2

Spécifications européennes des carburants pour 2000 et 2005

European specifications for motor fuels in 2000 and 2005

\begin{tabular}{l|c|c}
\hline \multirow{2}{*}{ Gazole } & \multicolumn{2}{|c}{ Limites 2000 } \\
\cline { 2 - 3 } & minimum & maximum \\
\hline Indice de cétane & 51 & 845 \\
\hline Densité à $15^{\circ} \mathrm{C}$ en $\mathrm{kg} / \mathrm{m}^{3}$ & & 360 \\
\hline $\begin{array}{l}\text { Distillation : } \\
\text { point } 95 \% \text { en }{ }^{\circ} \mathrm{C}\end{array}$ & & 11 \\
\hline Poly-aromatiques $(\%$ poids) & & 350 \\
\hline Soufre en $\mathrm{mg} / \mathrm{kg}$ & & \\
\hline \multicolumn{2}{|c}{ Limites 2005} \\
\hline
\end{tabular}

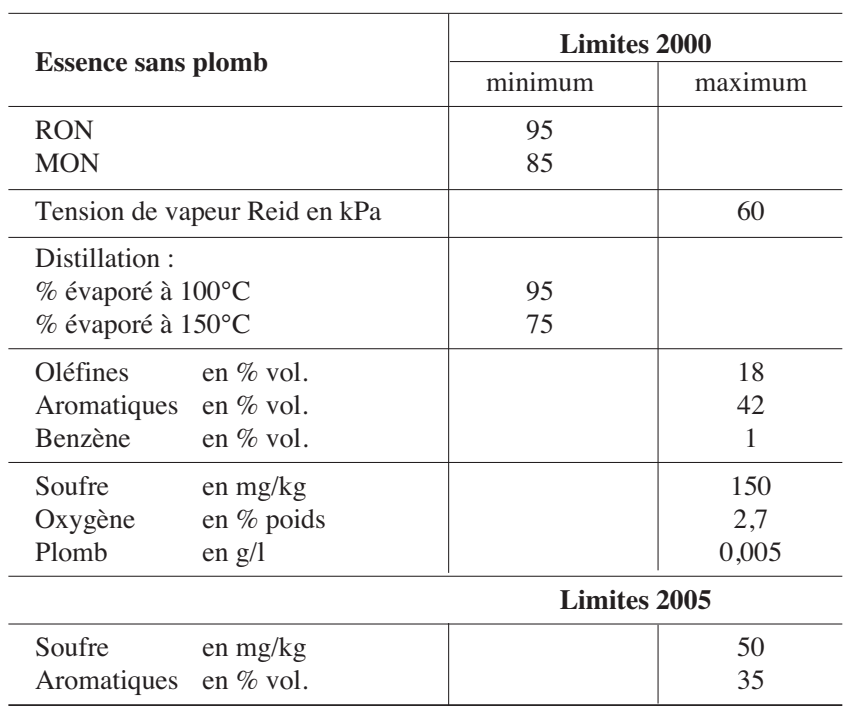


à celles proposées, en se fondant sur des critères de coût/efficacité, par le conseil des ministres en juin 1997 et non à celles, plus sévères, évoquées par le Parlement européen.

Les spécifications 2000 semblent donc pouvoir être satisfaites par la plupart des raffineries européennes au prix d'investissements modérés. En revanche, les normes 2005, plus sévères $a$ priori, pourraient conduire à des investissements importants (dès 2002) dans la quasi-totalité des raffineries européennes. L'évaluation finale, au-delà de la réduction de la teneur en soufre des carburants, dépendra des résultats du programme Auto-oil II attendus pour la fin 1999. Il est clair que pour certaines raffineries, l'alternative pourrait être de ne pas procéder à ces investissements et d'attendre l'entrée en vigueur des spécifications pour transformer leur site en dépôt et limiter ainsi le coût de fermeture.

Rappelons enfin que les raffineries devront également faire face, à ces horizons, à un renforcement marqué de leurs propres émissions $\left(\mathrm{SO}_{2}, \mathrm{NO}_{\mathrm{x}}\right.$, etc.) et à une diminution de la demande en fioul à haute teneur en soufre.

\section{CONCLUSION}

La situation mondiale du raffinage est encore fragile et les perspectives incertaines. Les marges nettes sont basses bien que les coûts aient diminué. En Europe, la perspective de normes 2005 relativement sévères, notamment pour le gazole (indice de cétane minimum de 54 voire plus, teneur en poly-aromatiques inférieures à $5 \%$ ), devraient conduire à des investissements importants. Sur la période, les raffineurs devront également faire face à une baisse de la demande en fioul lourd à haute teneur en soufre et à une réduction de leurs propres émissions. Dans ces conditions, des sites de raffinage pourraient se transformer en dépôt pour limiter les coûts de fermeture. Mais, à notre sens, une réduction de capacité trop importante en Europe ouvrirait la porte aux importations, en particulier du golfe Arabo-Persique où les énormes capacités d'exportation font face à un marché asiatique déclinant. 